\title{
Maddalena exploratory adit: feedback on hydrogeological and geothermal aspects
}

\section{Galleria geognostica La Maddalena: ritorno d'esperienza sugli aspetti idrogeologici e geotermici}

\author{
Antonio Dematteis, Piergiuseppe Gilli, Maria Elena Parisi, Lorenzo Ferrero, Fabio Furno
}

\begin{abstract}
Riassunto: La galleria geognostica La Maddalena $(7,5 \mathrm{~km}$ di lunghezza, diametro di scavo $6,3 \mathrm{~m}$ ) forma parte del progetto internazionale del nuovo collegamento ferroviario Torino-Lione, sul versante italiano del progetto, nel Comune di Chiomonte (TO). Tra le indagini geognostiche che vengono sistematicamente eseguite durante lo scavo vi sono misure di portate dell'acqua drenata, misure di temperatura dell'acqua e dell'ammasso roccioso. Il presente articolo analizza i dati registrati a tutto Aprile 2016, al fine di verificare i modelli previsionali geotermico ed idrogeologico e valutare il potenziale energetico delle venute di acqua calda che si prevede saranno intercettate dallo scavo del tunnel di base. I dati raccolti avvalorano la possibilità di una gestione sostenibile delle acque sotterranee e del geoscambio termico.
\end{abstract}

Parole chiave: Torino-Lione, galleria geognostica Maddalena, acque sotterranee, energia geotermica.

Keywords: Turin-Lyon, Maddalena exploratory adit, groundwater flow; geothermal energy.

Fabio FURNO 奉”

GEODATA Engineering S.p.A

Corso Bolzano 14, 10121 Turin, Italy.

Tel: +39.011.5810611 - Fax: +39 011.597440

E-mail: fabiofurno90@gmail.com

Antonio DEMATTEIS

Lorenzo FERRERO

GEODATA Engineering S.p.A

Piergiuseppe GILLI

Maria Elena PARISI

Tunnel Euralpin Lyon Turin SAS, Turin, Italy

Ricevuto/Received: 16 May 2016; Accettato/Accepted: 23 September 2016 Pubblicato online/Published online: 20 October 2016

This is an open access article under the CC BY-NC-ND license:

http://creativecommons.org/licenses/by-nc-nd/4.0/

(C) Associazione Acque Sotterranee 2016

\begin{abstract}
The $7.5 \mathrm{~km}$ long and 6,3 $\mathrm{m}$ diameter La Maddalena pilot tunnel is part of the international project of the new high speed rail link between Turin and Lyon, on the Italian side of the project (Chiomonte, TO). Among the geological surveys that are systematically performed during the excavation of the pilot tunnel, groundwater inflow and temperature of water and rock mass are measured. This paper analyses the recorded data to April 2016 in order to verify the hydrogeological and geothermal forecasting models and assess the energy potential of the inflows that are expected to be intercepted by the future base tunnel excavation. The collected data confirm the possibility of a sustainable management of groundwater and heat geoexchange.
\end{abstract}

\section{Introduzione}

Scopo di questo studio è l'analisi dei dati di temperatura e di portata di acqua emunta registrati durante lo scavo della galleria geognostica la Maddalena al fine di verificare le previsioni effettuate nel corso del progetto definitivo e valutare in maniera concreta la possibilità di uno sfruttamento delle risorse rese disponibili dallo scavo al fine di scambio termico. La galleria geognostica La Maddalena è propedeutica alla progettazione del tunnel di base del nuovo collegamento ferroviario Torino-Lione. Il progetto prevede tre discenderie in territorio francese già realizzate e due gallerie geognostiche, una in Francia i cui lavori sono iniziati alla fine del 2014 e quella de La Maddalena in territorio italiano con inizio scavo avvenuto a gennaio 2013. La Maddalena ha il suo imbocco nel comune di Chiomonte, circa $60 \mathrm{~km}$ ad Ovest di Torino, ha una lunghezza totale di $7500 \mathrm{~m}$ e un diametro di scavo pari a $6.3 \mathrm{~m}$. La pendenza nei primi $1500 \mathrm{~m}$ è verso l'alto, per facilitare l'evacuazione per gravità dell'acqua all'esterno, i successivi $2600 \mathrm{~m}$ sono inclinati verso il basso e la parte restante nuovamente verso l'alto. A partire dalla PK 3+600 lo scavo procede tra le due canne del tunnel di base, in direzione parallela ad esse, e ad una quota di poco superiore (Fig. 1).

Lo scavo dei primi $198 \mathrm{~m}$ è stato realizzato con metodo tradizionale, mentre la restante parte con scavo meccanizzato mediante una TBM aperta (Open Tunnel Boring Machine). Al momento della redazione di questo articolo (27/04/2016) lo scavo si trova alla progressiva chilometrica 4+776 (PK 4+776), quindi già in asse al tunnel di base. L'avanzamento dello scavo può essere monitorato attraverso il sito ufficiale TELT (Tunnel Euralpin Lyon Turin): http://www.telt-sas.com.

In fase di progetto del Tunnel di Base Torino Lione sono state proposte previsioni circa la temperatura dell'ammasso roccioso e le caratteristiche di quantità, qualità e temperatura 
delle acque, al fine di prevedere l'influenza che essi potranno avere sull'ambiente di lavoro durante lo scavo ed in fase di esercizio della galleria ferroviaria. Inoltre, queste valutazioni sono servite per individuare le possibilità di valorizzazione della risorsa idrica e termica del Tunnel di Base. E questo tenendo conto della forte influenza che, in ambiente alpino, il flusso idrico sotterraneo apporta al gradiente geotermico nel primo chilometro di profondità (Marechal, 2000; Jaboyedoff \& Pastorelli, 2003). I tunnel alpini come quelli del Gottardo, Ricken, Lötschberg sono esempi di sfruttamento delle venute d'acqua calda (Rybach, 2003). Le misure idrogeologiche e geotermiche nella galleria sono quindi indagini fondamentali per la corretta progettazione ed esecuzione dello scavo profondo del Tunnel di Base.

\section{Modello geologico di riferimento}

La galleria geognostica La Maddalena attraversa l'Unità di Ambin, ed in particolare il Complesso d'Ambin, costituito da gneiss leucocratici, ed il Complesso di Clarea, costituito da micascisti e gneiss (Polino et al. 2002). Nella Fig. 2 è mostrato il profilo geologico della galleria.

In questo settore l'Unità di Ambin è caratterizzata da una scistosità pervasiva immergente principalmente verso SE con angoli d'inclinazione tra $50^{\circ}$ e $75^{\circ}$. Le giaciture delle super- fici di scistosità indicano la presenza di pieghe asimmetriche aperte, con fianchi lunghi immergenti verso SE o S - SW e fianchi corti immergenti verso i quadranti opposti. Per quanto riguarda l'assetto strutturale fragile, sono presenti zone di faglia principali e fasce di intensa fratturazione con direzione NE-SW e inclinazione subverticale. Si riconoscono, inoltre, due sistemi di giunti ricorrenti, con persistenze e spaziature medie plurimetriche: uno con direzione E-W e inclinazione variabile da 60 a $70^{\circ}$, il secondo, ortogonale al precedente, ha direzione $\mathrm{N}-\mathrm{S}$ e valori di inclinazione di circa $80^{\circ}$. Il sistema di deformazione più importante a scala regionale, soprattutto nei confronti delle condizioni idrogeologiche nella galleria, è rappresentato da un insieme di faglie e fratture subverticali o fortemente immergenti verso SSE, con direzione $\mathrm{N} 60-70^{\circ} \mathrm{E}$. Il sistema è esteso all'intero versante sinistro della media ed alta Valle di Susa ed è parallelo all'asse vallivo principale e al contatto sudorientale tra l'unità di Ambin e la Zona Piemontese; può essere seguito nelle immagini aeree dalla zona del M. Seguret fino alla Val Cenischia e alla testata della Val Clarea (Cadoppi, Martinotti, Università di Torino- Dipartimento di Scienze della Terra, unpublished 2005) ed è quindi stato denominato "Zona di deformazione Seguret - col Clapier" o ZSC. La sua persistenza è dell'ordine dei $10-15 \mathrm{Km}$, la potenza complessiva è di 2000-5000 m circa. Lungo il profilo

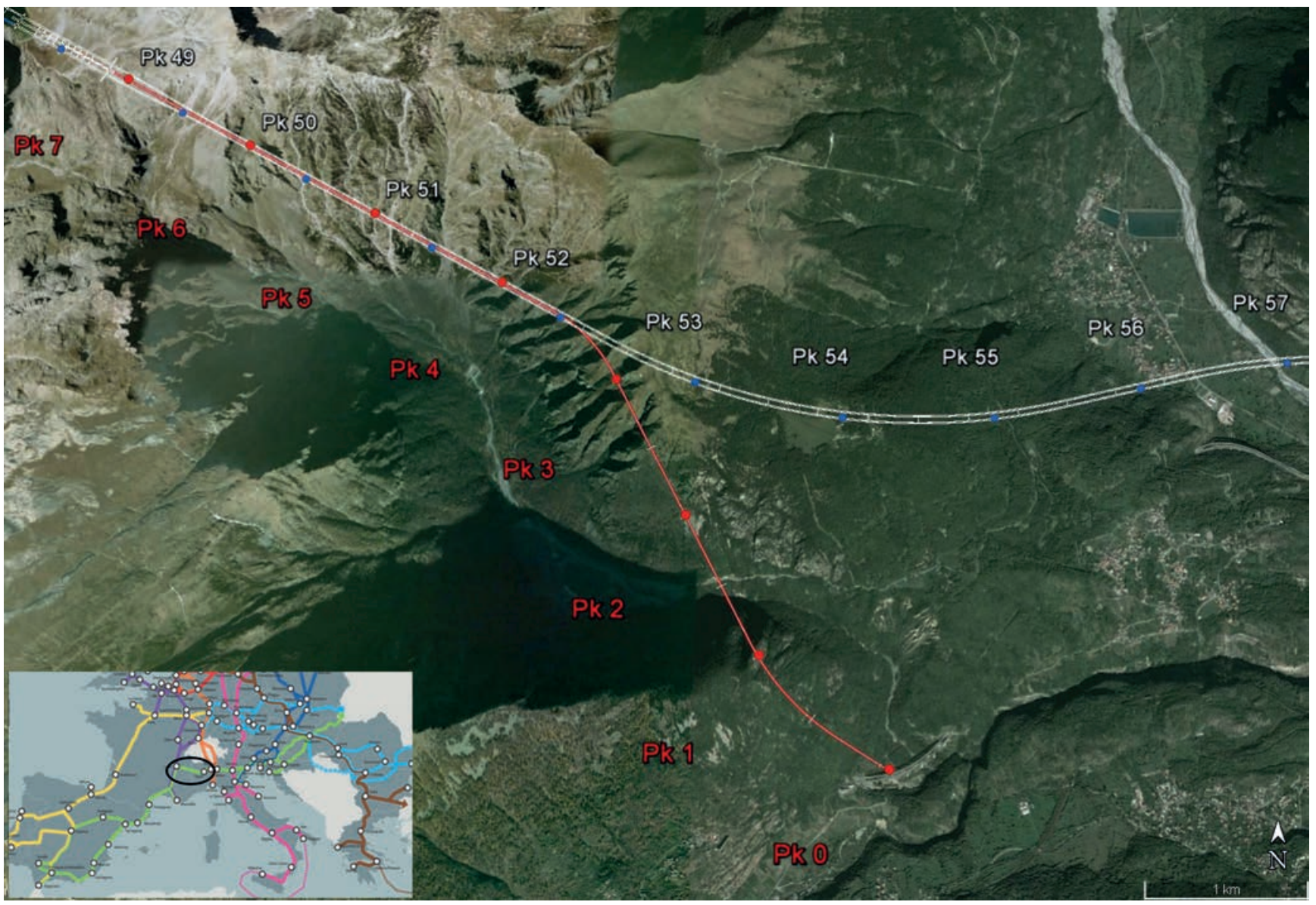

Fig. 1 - Ubicazione galleria geognostica La Maddalena, con le progressive chilometriche (PK) in rosso, ed il Tunnel di Base del nuovo collegamento ferroviario Torino-Lione, con le PK in bianco (su foto aerea ${ }^{\circledR}$ GoogleEarth).

Fig. 1 - Location of the Maddalena geognostic gallery, with progressive kilometers (PK) in red, and the tunnel of the new rail link. between Turin and Lyon, with white PK. 
geologico della galleria (Fig. 2) tale sistema di faglie e zone di intensa fratturazione è indicato tra le PK 4+500 e PK 7+000 circa. Diverse informazioni sulle sue caratteristiche sono basate sui dati di sottosuolo derivanti dallo scavo della galleria di derivazione dell'impianto idroelettrico di Pont Ventoux (AEM Torino).

Sul profilo geologico della Fig. 2 le linee tratteggiate a più basso angolo indicano la giacitura della scistosità principale.

Gli gneiss e i micascisti del Complesso di Clarea e d'Ambin sono stati raggruppati in un unico Complesso Idrogeologico, al quale è attribuita una permeabilità a grande scala di grado basso, legata alla presenza della citata fratturazione e talvolta di zone di faglia. La permeabilità secondaria per fratturazione è stata valutata tramite prove in foro, eseguite in tre sondaggi profondi lungo l'asse della galleria, che hanno indicato valori compresi tra 5E10-8 e 5E10-7 m/s. In galleria, le venute d'acqua importanti si sono verificate in corrispondenza dell'intersezione di faglie e bande di fratturazione più intensa, che normalmente hanno uno spessore variabile tra qualche metro e qualche decina di metri. Queste venute sono di tipo puntuale con portate che variano nel tempo in funzione del carico idraulico e del loro grado di connessione con le zone di ricarica.

\section{Materiali e Metodi Misure di temperatura e portata}

Nel corso dello scavo del cunicolo geognostico vengono sistematicamente effettuate misure di portata delle venute d'acqua e di temperatura della roccia e dell'acqua. La portata totale di acqua drenata dalla galleria è monitorata all'esterno, presso l'impianto di trattamento delle acque mediante misuratore elettromagnetico. Le misure di temperatura della roccia sono realizzate mediante termoresistenze (precisione $+/-0.5^{\circ} \mathrm{C}$ ) posizionate in fori di $1,5 \mathrm{~m}$ di profondità perforati sui paramenti della galleria ogni $50 \mathrm{~m}$ di avanzamento dello scavo. Ogni $500 \mathrm{~m}$ di galleria uno di questi fori viene equipaggiato con stazione fissa, al fine di poter garantire la misura a lungo termine, garantendo l'accesso alla stazione attraverso una finestra nel rivestimento definito. Le altre stazioni inter- medie, temporanee, garantiscono misure per un intervallo temporale sufficiente ad ottenere condizioni stazionarie della temperatura, successivamente vengono rimosse e coperte dal rivestimento definitivo della galleria (Fig. 3). La misura della temperatura delle acque è eseguita con termometri elettronici, con una precisione di $+/-0.5^{\circ} \mathrm{C}$.
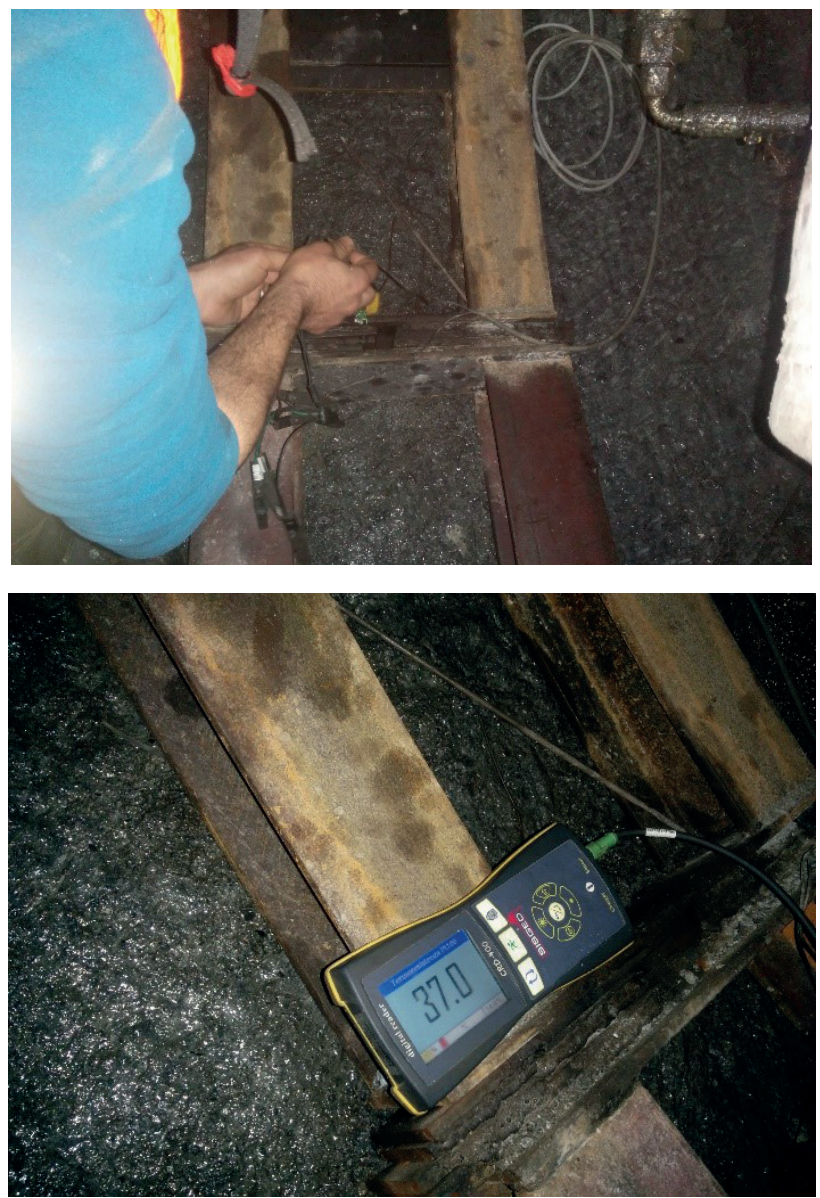

Fig. 3 - Stazioni di misura della temperatura in galleria, stazione fissa a sinistra, stazione temporanea a destra.

Fig. 3 -Measuring stations of temperature in the tunnel, fixed station on the left, temporary station on the right.

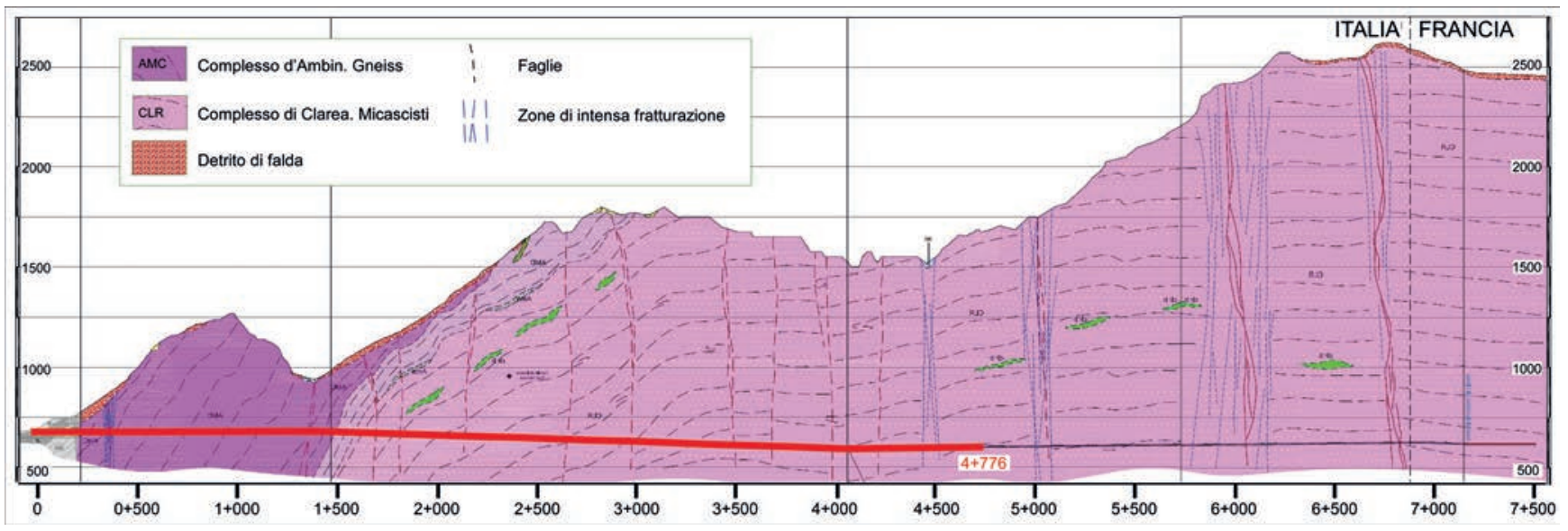

Fig. 2 - Profilo geologico del Cunicolo esplorativo La Maddalena. Il tratto rosso lungo il tracciato indica la parte già scavata. Tratto dal profilo geologico di progetto. Fig. 2 - Geological profile of the Maddalena exploratory adit. The red line along the path indicates the part already excavated. From the geological project profile. 
Nella Fig. 4 sono mostrati i valori di temperatura della roccia misurati lungo la galleria e l'andamento delle temperature che sarebbero ipotizzabili applicando un gradiente geotermico costante nel massiccio d'Ambin, assunto arbitrariamente pari a $25^{\circ}$ e $30^{\circ} \mathrm{C} / \mathrm{km}$, solo con lo scopo di confrontare i dati. Si può notare come fino alla PK 3+000 circa la temperatura aumenti gradualmente e regolarmente all'approfondirsi della galleria, indipendentemente dal sottopasso del torrente Clarea che occorre alla PK 1+400 circa. Successivamente, a partire dalla PK 3+000 il gradiente di temperatura aumenta, pur con una lieve diminuzione della copertura, fino alla PK $4+000$ circa. Più avanti la temperatura della roccia nella galleria scende bruscamente, fino a $36^{\circ} \mathrm{C}$ alla progressiva 4100 $\mathrm{m}$ circa. Se da un lato per spiegare queste variazioni occorre considerare l'evidente effetto tridimensionale della copertura sulla temperatura a quota tunnel (Fig. 1), d'altro canto queste anomalie possono essere messe in relazione anche ad altra fonte, di tipo tettonico. Come si vede nella pianta di Fig. 1, a partire dalla progressiva $3+000$ la galleria entra nella parte più interna e profonda del massiccio di Ambin. Il profilo geologico (Fig. 2) indica in questo tratto la presenza di faglie minori, ad alto angolo, che evidentemente non hanno un ruolo importante dal punto di vista idrogeologico, prova ne è la modesta quantità di acqua che viene drenata dalla galleria, e la modesta estensione della zona di ricarica in superficie. Dopo la progressiva $4+500$ il profilo geologico (Fig. 2) indica la presenza di ripetute fasce di intensa fratturazione, corrispondenti al sistema ZSC menzionato sopra, che data la sua persistenza pluri-chilometrica potrebbe essere sede di celle di flusso profonde, le cui acque nella parte di risalita potrebbero generare locali aumenti della temperatura del massiccio.

Nella Fig. 5 sono riportati i valori di temperatura della roccia in funzione della profondità della misura, a prescindere dalla progressiva. È possibile distinguere tre zone con andamenti omogenei: i) fino alla profondità di circa $600 \mathrm{~m}$, ed in particolare per temperature inferiori a $20^{\circ} \mathrm{C}$, i dati paiono dispersi, senza indicare un chiaro gradiente geotermico; segue poi ii) una zona intermedia, tra 600 e $800 \mathrm{~m}$ di profondità, dove il gradiente termico è regolare, relativamente basso, pari a circa $15,5^{\circ} \mathrm{C} / \mathrm{km}$, ed infine, iii) a partire dagli $800 \mathrm{~m}$ di profondità, e fino alle massime coperture raggiunte, pari a 1200 $\mathrm{m}$, la temperatura della roccia nel tunnel è assai variabile, a prescindere dalla profondità. Le temperature più elevate, oltre i $40{ }^{\circ} \mathrm{C}$ e fino a $50^{\circ} \mathrm{C}$, sono riscontrate a profondità comprese tra 900 e 1100 m, che corrispondono alle PK 3+700 e 4+100 circa (Fig. 4).

Questa dispersione dei punti del grafico di Fig. 5, special-

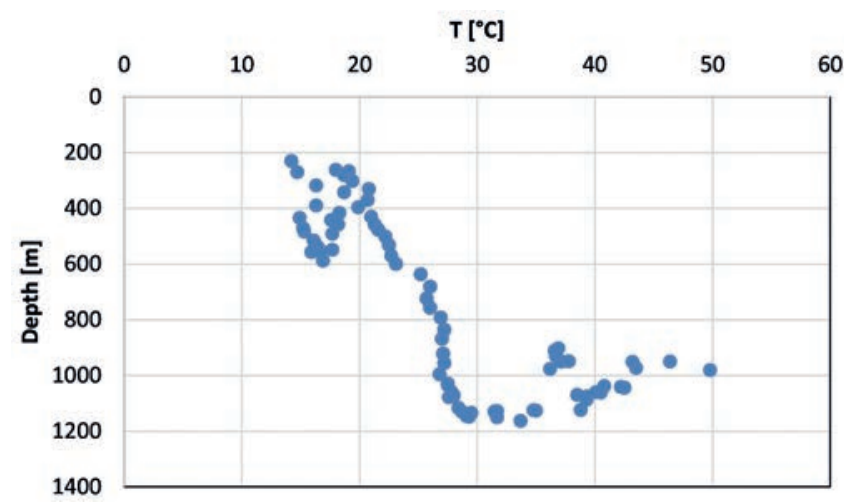

Fig. 5 - Valori di temperatura della roccia misurati nel tunnel in funzione della profondità in quel punto.

Fig. 5 - Values of measured temperature of the rock in the tunnel compared with coverage.

mente nelle zone (1) e (3), è probabilmente da mettere in relazione a due fattori che agiscono simultaneamente tra loro: l'effetto di raffreddamento dovuto alla topografia acclive dei versanti (nei settori dove la galleria si trova a quota superirioe a quella del livello idrogeologico di base, che in questo settore è dato dal torrente Dora Riparia) e la presenza di sistemi di flusso idrico con circolazione rapida e discendente, fredda, all'interno dei sistemi di più intensa fratturazione intercettati

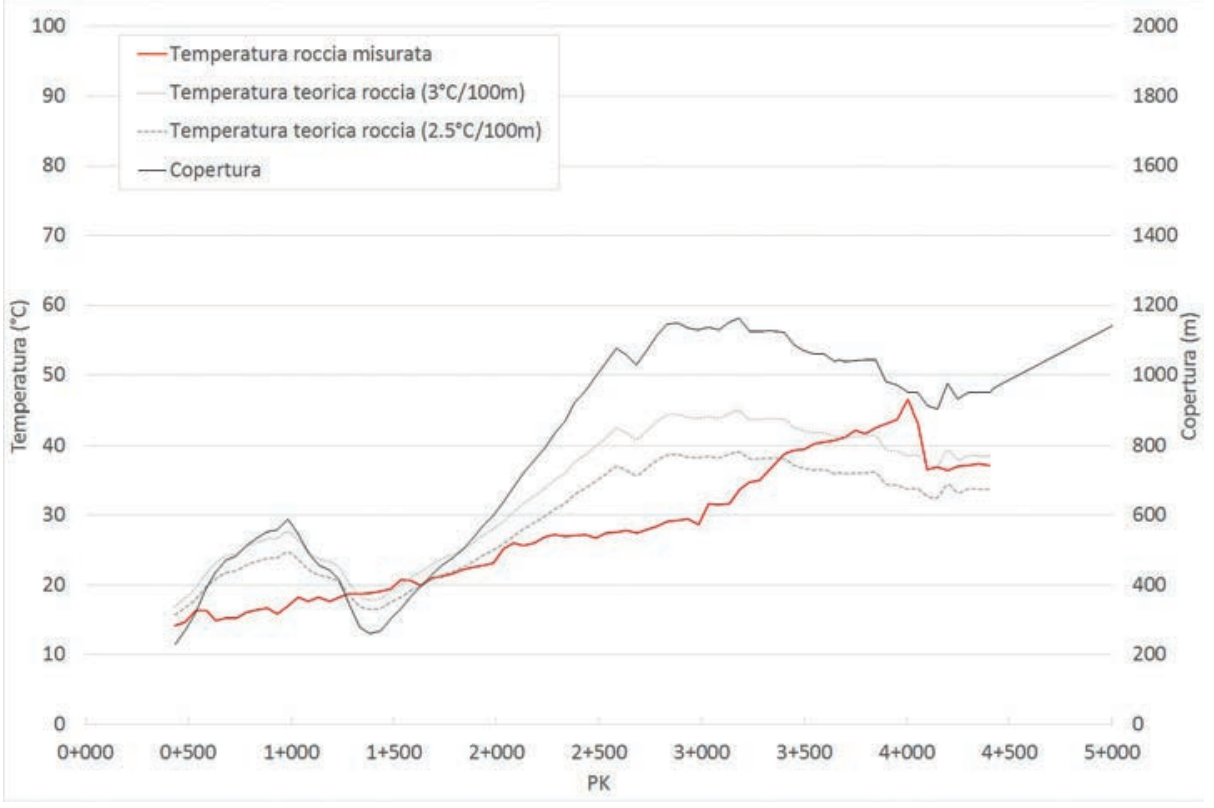

Fig. 4 - Andamento della temperatura dell'ammasso roccioso lungo la galleria geognostica. Temperatura misurata (linea rossa) confrontata con stime di temperatura effettuate assumendo gradienti geotermici costanti pari a 25 e $30^{\circ} \mathrm{C} / \mathrm{km}$.

Fig. 4 - Rock mass temperature along the tunnel surveys. Measured temperature (red line) compared with temperature estimation assuming constant geothermal gradient of 25 and $30^{\circ} \mathrm{C} / \mathrm{km}$. 
dallo scavo. Al fine di motivare questi differenti andamenti, nel grafico di Fig. 6 si analizzano le temperature della roccia a confronto con le venute d'acqua nel tunnel, in modo da valutare il contributo convettivo al trasporto di calore all'interno dell'ammasso. L'andamento delle misurazioni è stato diviso per chiarezza espositiva in tre tratti (Tab. 1 e Fig. 4):

- il primo tratto si estende tra le PK $0+500$ e $1+500$, in cui vi è variabilità della copertura (PK $1+000$, profondità $600 \mathrm{~m}$, PK $1+500$, profondità $300 \mathrm{~m}$ ). All'interno di questo tratto le portate subiscono forti oscillazioni. Questa configurazione ha un effetto determinante sulla temperatura, che non risulta essere correlata alla copertura, come dovrebbe essere considerando un gradiente medio costante;

- il secondo tratto si estende tra le PK $1+500$ e $2+800$, in cui è possibile evidenziare un aumento quasi lineare della copertura. Ipotizzando un gradiente geotermico costante il graduale aumento della temperatura della roccia dovrebbe essere legato al progressivo approfondimento dello scavo, ma analizzando i dati è possibile osservare come il tratto in esame sia contraddistinto da una progressiva crescita della portata cumulata drenata dal tunnel. In particolare, sono registrate quattro venute puntuali (PK da $1+700$ a $1+900$, PK 1+930, PK 2+220, PK da $2+625$ a $2+765)$, che potrebbero essere la causa della moderata crescita di temperatura con la profondità;

- il terzo tratto si estende dalla PK 2+800 alla 4+400, in cui la profondità di scavo dopo un breve tratto costante pari alla massima copertura raggiunta $(1150 \mathrm{~m})$ tende a diminuire. All'interno di questo tratto la portata diminuisce progressivamente, per effetto dell'assenza di venute d'acqua rilevanti in galleria, e simultaneamente la temperatura della roccia cresce, notevolmente, fino alla PK 4+000, per poi diminuire bruscamente di circa $14^{\circ} \mathrm{C}$ (da 50 a $36^{\circ} \mathrm{C}$ ) in $200 \mathrm{~m}$. Laumento della temperatura è da mettere in relazione al diminuito flusso di acque sotterranee verso la galleria. Analizzando la Fig. 4 si nota che, all'interno di questo tratto, le temperature registrate sono più alte di quelle calcolate considerando un gradiente geotermico costante. Questa anomalia non è completamente giustificabile dal ridotto flusso di acque fredde discendenti, che potrebbe giustificare il riscaldamento anomalo; essa potrebbe essere legata ad una struttura geologica che favorisce un flusso rimontante di acque con temperature più elevate. Questa resta pertanto un'ipotesi da verificare.

Tab. 1 - Suddivisione della galleria in tratti omogenei dal punto di vista idrogeologico e geotermico.

Tab. 1 -Tunnel division into homogeneous sections from the hydrogeological and geothermal point of view.

\begin{tabular}{|l|c|c|c|c|c|c|c|}
\cline { 2 - 7 } \multicolumn{1}{c|}{} & \multicolumn{3}{c|}{$\begin{array}{c}\text { Section I } \\
\text { (Tratto I) }\end{array}$} & \multicolumn{2}{c|}{$\begin{array}{c}\text { Section II } \\
\text { (Tratto II) }\end{array}$} & \multicolumn{2}{c|}{$\begin{array}{c}\text { Section III } \\
\text { (Tratto III) }\end{array}$} \\
\hline PK & 500 & 1000 & 1500 & 1500 & 2800 & 2800 & 4400 \\
\hline $\begin{array}{l}\text { Depth } \\
\text { (Profondità) }\end{array}$ & 300 & 600 & 300 & 300 & 1150 & 1150 & 950 \\
\hline $\begin{array}{l}\text { Flow rate } \\
\text { (Portata) }\end{array}$ & \multicolumn{3}{|c|}{$\begin{array}{c}\text { Higly variable } \\
\text { (Molto variabile) }\end{array}$} & $\begin{array}{c}\text { Increasing } \\
\text { (In aumento) }\end{array}$ & $\begin{array}{c}\text { Decreasing } \\
\text { (In diminuzione) }\end{array}$ \\
\hline
\end{tabular}

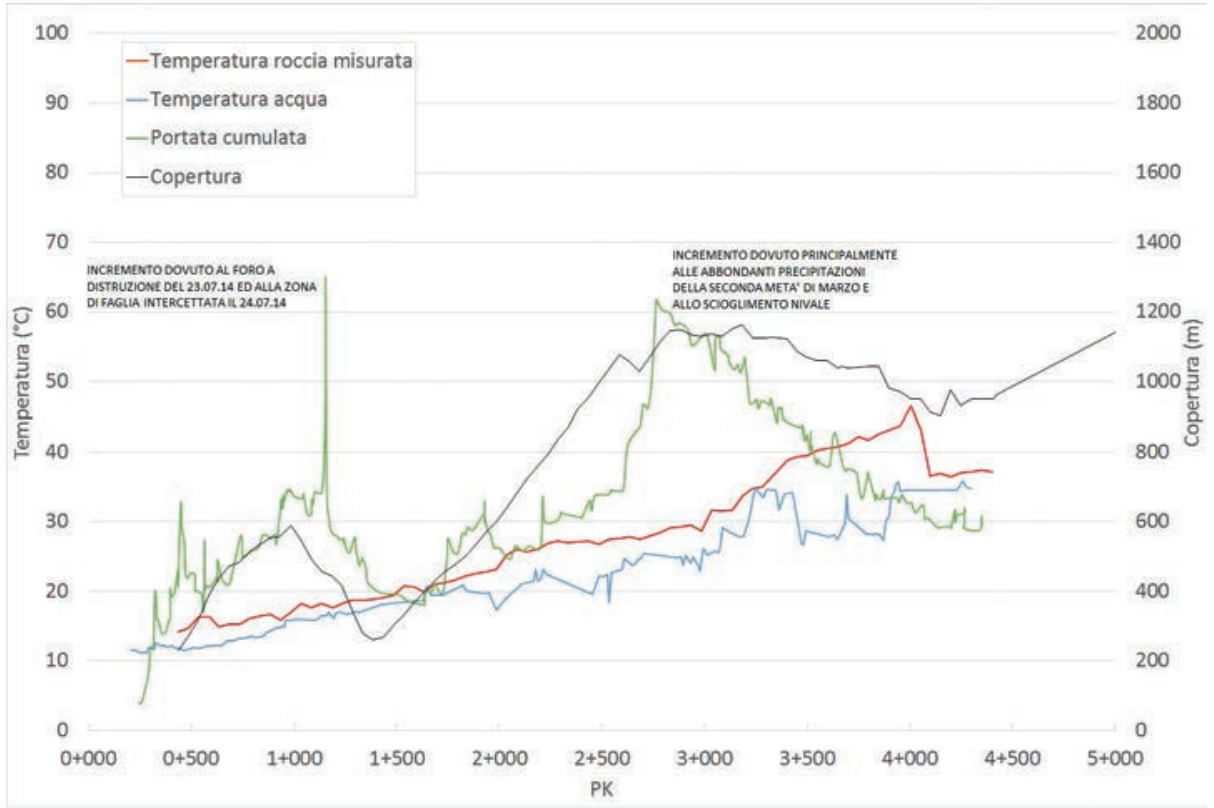

Fig. 6 - Andamento delle temperature della roccia e delle portate di acqua drenate dalla galleria.

Fig. 6 - Trend of rock temperatures and flow rates of drained water from the gallery. 


\section{Verifica delle ipotesi di progetto Portate}

La Fig. 7 mostra il confronto tra le portate previste in fase di progetto e le portate registrate in corso di scavo della galleria geognostica. Nella prima parte dello scavo vengono attraversati depositi quaternari di versante, non consolidati e permeabili per porosità. Successivamente, e per tutta la galleria, le venute d'acqua si generano in corrispondenza di faglie e giunti principali. Le portate registrate risultano dello stesso ordine di grandezza del minimo previsto prima dell'inizio degli scavi, pur essendo inferiori, circa la metà. Queste portate non sono stabilizzate e lo scavo non è ancora terminato, ciononostante esse risultano dell'ordine dei 30 1/s, quantità che giustifica l'ipotesi di una valorizzazione all'esterno delle acque del tunnel di base, al fine di non disperdere la risorsa naturale.

\section{Temperatura}

Per quanto riguarda il tunnel di base, per il quale si sta realizzando la galleria geognostica, il modello geotermico di progetto fu implementato attraverso numerose campagne di sondaggi geognostici, log termici fino alla quota del tunnel e modellazioni. Questi studi hanno permesso di definire un profilo previsionale di temperature a quota tunnel. La previsione indica una zona con estensione di circa $8 \mathrm{~km}$, tra le PK 41 e 52 , con temperatura superiore a $40^{\circ} \mathrm{C}$, con una temperatura massima di $47-48^{\circ} \mathrm{C}$ raggiunta tra la $\mathrm{PK} 44$ e 48 . La galleria La Maddalena, alla sua PK 3+470 circa raggiunge il Tunnel di Base, alla sua PK 52. La temperatura della roccia registrata nel cunicolo La Maddalena alla PK 3+450 è pari a $39^{\circ} \mathrm{C}$ (Fig. 6), che è in linea con la temperatura della roccia prevista per il tunnel di base (PK 52), che è compresa tra 35 e $40^{\circ} \mathrm{C}$.

\section{Gestione sostenibile delle acque in galleria}

Il progetto del tunnel di base prevede di collettare all'esterno l'acqua di galleria, attraverso tubazioni ben isolate al fine di ridurre la dispersione termica (Fig. 8). Per quanto riguarda la possibilità di drenare le acque sotterranee del massiccio, le nuove normative per il rispetto ambientale impongono una minimizzazione dell'impatto idrogeologico dello scavo, per questo motivo la soluzione adottata per il tunnel di base è stata quella di un rivestimento totalmente impermeabile ove le pressioni idrauliche all'estradosso del rivestimento non superino i 10 bar (per ragioni tecnologiche l'impermeabilizzazione totale è possibile solo per pressioni inferiori). Nelle zone di alta copertura, dove si prevede che le pressioni d'acqua a quota tunnel possano essere superiori, la galleria è drenante. Il sistema di collettamento delle acque all'interno della galleria permette di separare le acque di piattaforma, potenzialmente inquinate, da quelle naturali del massiccio, di buona qualità, che vengono ulteriormente separate in base alla loro potenzialità di riutilizzo all'esterno. All'esterno, un impianto con pompa di calore acqua-acqua permetterà l'estrazione del calore dall'acqua di galleria. I dati di temperatura e di portate presentati sopra, raccolti nella galleria geognostica, confermano l'adeguatezza delle ipotesi fatte per la gestione delle acque nel Tunnel di Base.

\section{Recupero dell'energia geotermica}

Lo sfruttamento del calore geotermico misurato nella galleria geognostica può teoricamente essere effettuato in due modi, già applicati in altre gallerie: attraverso scambiatori di calore, che inseriti nel rivestimento permettano di estrarre il calore della roccia, oppure mediante pompe di calore che utilizzano l'acqua intercettata dallo scavo, più calda rispetto a quella esterna, per sfruttare la differenza termica.
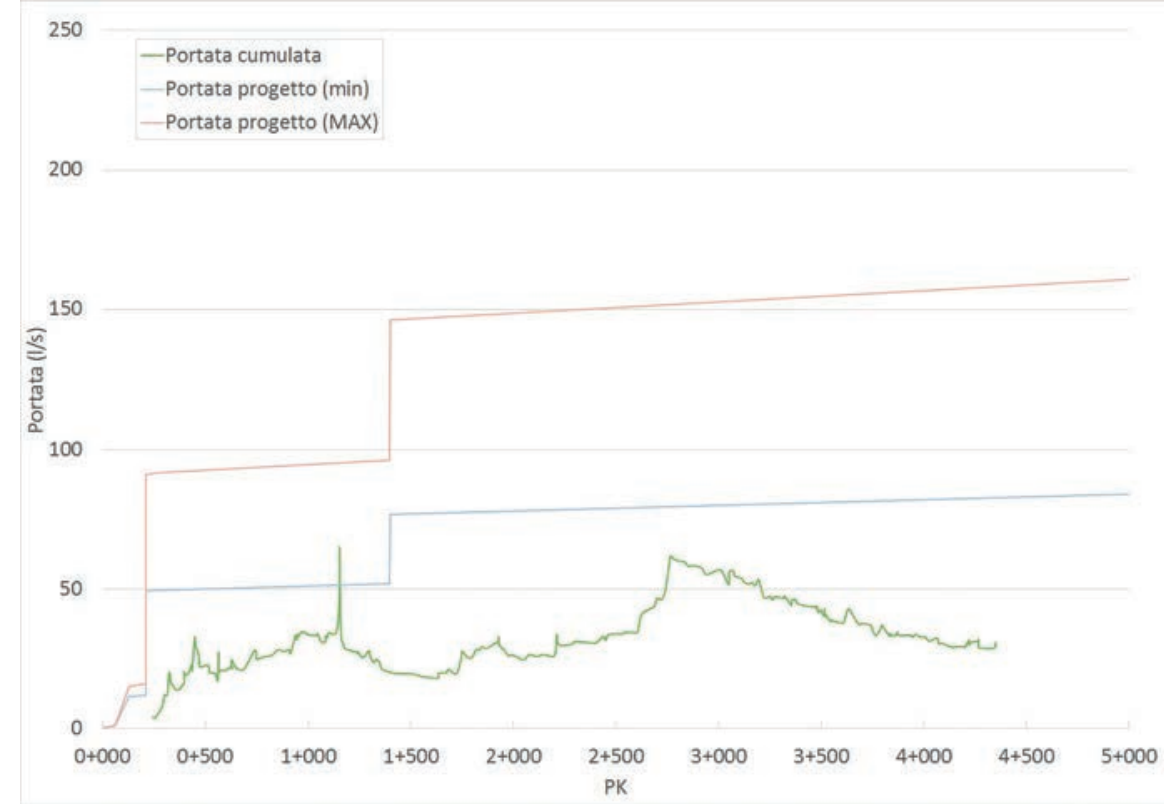

Fig. 7 - Confronto tra portate cumulate
misurate nella galleria geognostica e por-
tate minime e massime previste in fase di
progetto.
Fig. 7 - Comparison between cumulative flow
rates measured in surveys gallery and minimum
and maximum flow rates provided envisaged in
the design phase. 


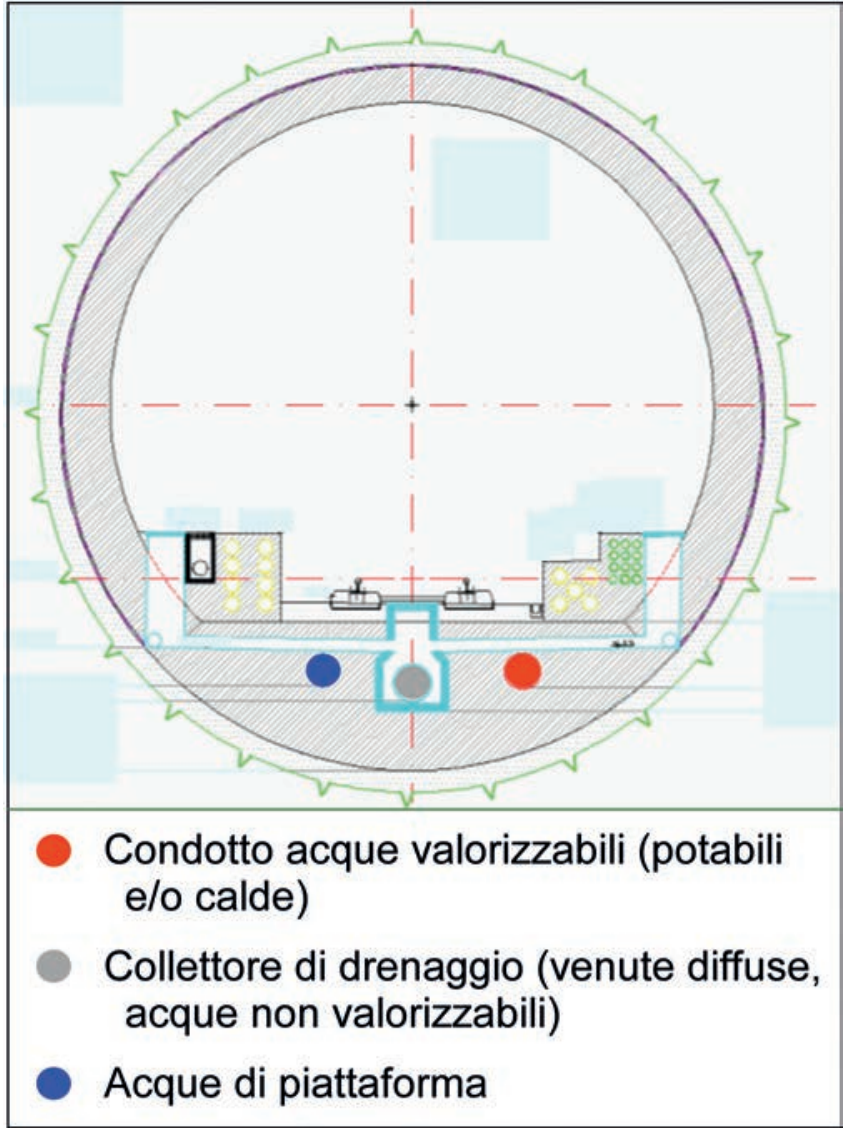

Fig. 8 - Sistema di drenaggio e gestione delle acque nel Tunnel di Base Torino Lione.

Fig. 8 - Drainage system and water management in the Base Tunnel Turin-Lyon.

Il primo tipo di sfruttamento è già stato sperimentato in altre gallerie, tramite l'installazione di scambiatori di calore all'interno del rivestimento del tunnel, dove il fluido scambiatore di calore scorre nei tubi incorporati nel calcestruzzo dei segmenti di rivestimento. Gli aspetti tecnologici di un impianto di questo tipo sono descritti per la galleria di Jenbach (Frodl et al. 2010 Franzius e Pralle 2011) e per la metropolitana di Torino (Barla \& Perino 2014, Barla et al. 2015). Questa ipotesi presenta, tuttavia, una forte limitante legata agli ingombri che l'impianto da adottare richiede (Furno et al. 2015, Soldo et al., 2016). In definitiva, l'esperienza dimostra che gli scambiatori di calore nel calcestruzzo di rivestimento della galleria risultano più vantaggiosi per tunnel metropolitani, i quali situandosi a bassa profondità ed in contesti densamente urbanizzati, hanno il vantaggio di avere un'utenza diffusa a pochi metri dal tunnel, che permette di sfruttare brevi tratti di galleria e condurre più frequentemente e facilmente l'energia fuori dal tunnel. La possibilità di allestire impianti per settori di tunnel permette di limitare notevolmente gli ingombri dell'impianto di conduzione nei rivestimenti. Situazione decisamente opposta è quella de La Maddalena, che è una galleria profonda in ambiente montano poco popolato.

La seconda ipotesi, ovvero di condurre all'esterno le acque calde drenate nella galleria ed estrarre l'energia termica mediante pompe di calore acqua - acqua risulta in questo caso più fattibile.

In Tab. 2 sono riportate le temperature dell'acqua registrate al sollevamento dell'impianto di depurazione a monte delle torri di raffreddamento, il cui funzionamento è stato attivato nel mese di maggio del 2016 al fine di raffreddare l'acqua prima del suo utilizzo per i servizi del cantiere e della galleria e per diminuirne la temperatura allo scarico che dunque ha, a partire dal mese di maggio 2016, una temperatura inferiore a seguito del raffreddamento operato all'interno delle torri evaporative. Al punto di misura, l'acqua ha già subito variazioni di temperatura per il lungo tragitto nelle tubazioni a monte dell'impianto, in galleria e nei piazzali esterni, per lo scambio di calore con l'ambiente nelle vasche dell'impianto, per i ricicli di fanghi e spurghi dell'impianto.

I valori mensili sotto riportati, sono la media delle misure registrate relative a circa due giorni consecutivi, i dati sono registrati con cadenza di 15 minuti primi.

Considerando le temperature dell'acqua intercettata dallo scavo, effettuate nel corso dello scavo del tunnel geognostico (Tab. 2) è possibile stimare la temperatura media dell'acqua emunta e quindi stimare la potenza termica disponibile, mediante la seguente formula:

$$
P\left(W_{t}\right)=C \cdot Q \cdot \Delta T
$$

Dove $C$ è la capacità termica specifica dell'acqua, $Q$ è la portata del flusso d'acqua [L/s] stimata come portata cumulata e $\Delta T$ è la differenza di temperatura utilizzata ai fini del riscaldamento. Considerando un impianto con pompe di calore in serie, è possibile sfruttare una differenza tra temperatura dell'acqua in ingresso ed in uscita di $5^{\circ} \mathrm{C}$, ottenendo quindi una potenza termica pari a $874 \mathrm{~kW}$ (Tab. 3).

Tab. 3 -Calcolo potenza termica acqua emunta.

Tab. 3 - Water thermal power calculation.

\begin{tabular}{|c|c|c|c|c|}
\hline $\mathrm{C}[\mathrm{J} / \mathrm{lK}]$ & $\mathrm{T}_{0}\left[{ }^{\circ} \mathrm{C}\right]$ & $\mathrm{T}_{\text {media }}\left[{ }^{\circ} \mathrm{C}\right]$ & $\mathrm{Q}[1 / \mathrm{s}]$ & $\mathrm{P}[\mathrm{kW}]$ \\
\hline 4200 & 12 & 17.00 & 41.6 & 874 \\
\hline
\end{tabular}

Tab. 2 - Temperatura dell'acqua registrata nel corso dello scavo della galleria geognostica.

Tab. 2 - Water temperature recorded during the excavation of the Maddalena tunnel.

\begin{tabular}{|c|c|c|c|c|c|c|c|c|c|c|c|c|}
\hline & \multicolumn{11}{|c|}{ Temperature $\left({ }^{\circ} \mathrm{C}\right)$} & \multirow[b]{2}{*}{ December } \\
\hline Date & January & February & March & April & May & June & July & August & September & October & November & \\
\hline 2014 & - & - & 12.60 & 13.00 & 13.48 & 14.80 & 15.70 & 16.33 & 16.19 & 15.40 & 14.40 & 13.15 \\
\hline 2015 & 13.00 & 13.30 & 14.30 & 15.39 & 16.65 & 17.21 & 18.75 & 18.02 & 18.38 & 18.39 & 16.99 & 16.01 \\
\hline 2016 & 16.25 & 18.60 & 18.99 & 20.35 & 21.54 & 23.06 & 24.38 & 25.40 & - & - & - & - \\
\hline
\end{tabular}


Questo dato, anche se riferito ad una situazione transitoria, conferma l'effettiva potenzialità di geoscambio termico nel Massiccio d'Ambin. Al fine di valutare in maniera completa l'impatto dello sfruttamento delle acque provenienti dalla galleria resta tuttavia da individuare la struttura servita dall'impianto di climatizzazione ed il profilo energetico da essa richiesto. Il potenziale risulta essere superiore a quello stimato da LTF, in via preliminare nel 2013, pari a $870 \mathrm{~kW}$ per il riscaldamento della stazione di Susa.

\section{Discussione}

Lo scopo principale della galleria geognostica La Maddalena è quello di arricchire la conoscenza del massiccio d'Ambin, al fine di migliorare il progetto definitivo del tunnel di base Torino-Lione. Le misure di venute d'acqua e temperatura della roccia raccolte nella galleria geognostica permettono una prima verifica dei modelli previsionali ed una conferma della possibilità del utilizzo del geoscambio termico.

Le portate cumulate registrate in uscita dalla galleria geognostica risultano dell'ordine di 30 l/s, quantità che conferma la fattibilità di una valorizzazione all'esterno delle acque del tunnel di base, al fine di non disperdere la risorsa naturale.

Le misure effettuate nella tratta già scavata della galleria geognostica confermano il modello geotermico previsionale elaborato da LTF (Lyon Turin Ferroviaire) nel 2012 nell'ambito del Progetto Definitivo del Tunnel di Base (LTF SAS, unpublished 2012). Il gradiente geotermico del massiccio d'Ambin nelle parti corticali, con copertura inferiore a $600 \mathrm{~m}$ circa, è basso e molto variabile, essendo fortemente influenzato dalle aree di ricarica, che di fatto raffreddano il massiccio. Nelle parti più interne del massiccio il gradiente cresce, a causa di un diversa circolazione idrica, che risulta più concentrata in zone di faglia o canali di fratturazione più intensa, generalmente con andamento prossimo alla verticale. Nel tratto tra le PK 3+000 e 4+100 la galleria geognostica assume coperture comprese tra $900 \mathrm{~m}$ e $1100 \mathrm{~m}$ e si pone in asse al tunnel di base. In questo tratto della galleria il gradiente geotermico si attesta attorno a $22,2{ }^{\circ} \mathrm{C} / \mathrm{km}$ nei primi $900 \mathrm{~m}$, per poi decrescere bruscamente nei successivi $200 \mathrm{~m}$, dove le temperature si abbassano di $14^{\circ} \mathrm{C}$. Questa anomalia è probabilmente legata a sistemi di flusso localizzati in zone di maggiore permeabilità nel massiccio, rappresentati da zone di falgia o di intensa fratturazione. Questa ipotesi deve essere approfondita mediante analisi geologico strutturali idrogeochimiche ed isotopiche nella galleria.

\section{Conclusioni}

I dati raccolti avvalorano la possibilità di una gestione sostenibile delle venute d'acqua all'interno dello scavo e di uno sfruttamento termico delle acque drenate. La metodologia di sfruttamento proposta nell'ambito del progetto definitivo, attraverso l'utilizzo di pompe di calore, risulta essere la più vantaggiosa rispetto all'alternativa attraverso scambiatori di calore integrati nel rivestimento definitivo della galleria.

\section{BIBLIOGRAFIA}

Franzius JN, Pralle N, 2011. Turning segmental tunnels into sources of renewable energy. Proceedings of the ICE - Civil Engineering, 164(1): 35-40.

Frodl S, Franzius JN, Bartl T, 2010. Design and construction of the tunnel geothermal system in Jenbach. Geomechanics and Tunnelling 3, N. 5; 658-668.

Furno F, Barla M, Dematteis A, Lo Russo S, 2015. Methodological approach for sustainable management of water inflow and geothermal energy in tunnels. Acquesotterranee - Italina Journal of Groundwater: 37-43. DOI 10.7343/AS-119-15-0146.

Hufschmied P, Brunner A, 2010. The exploitation of warm water through the exemple of the Lötschberg Base Tunnel in Switzerland. Geomechanics and Tunnelling 5: 647-657

Jaboyedoff M, \& Pastorelli S, 2003. Perturbation of the heat flow by water circulation in a mountainous framework. Examples from the Swiss Alps. Eclogae Geologicae Helvetiae, 96: 37-47.

Marechal JC, 2000. Thermal inertia of Alpine massif shown by heat trnsport modelling: the large-scale Mont Blanc case study. Tracers and Modelling in Hydrogeology (proceedings of the TraM2000 Conference held at Liege, Begium May 2000. IAHS Publ. no. 262, 2000. Pp. 289-296.

Polino, R., Dela Pierre, F., Borghi, A., Carraro, F., Fioraso, G. \& Giardino, M., 2002. Carta geologica d'Italia alla scala 1:50.000, foglio 152-153 Bardonecchia e note illustrative " Hydrogeological Map of Italy-Scale 1:50.000 and illustrative notes". Regione Piemonte.

Rybach L, Wilhelm J, Goran H, 2003. Geothermal use of tunnel waters- a Swiss speciality. International Geothermal Conference Session 5, Reykjavì, Sept. 2003.

Soldo L, Dematteis A, Furno F, Barla M, 2016. Istanbul metro: a possible example of energy geostructure, SBE 2016, Towards postcarbon cities, Torino, 18-19 Febbraio. 